\title{
Qualitative Analysis of a Pest Management Gompertz Model with Interval State Feedback Impulsive Control
}

\author{
Yuan Tian, ${ }^{1}$ Zhantao Zhang, $^{1}$ and Kaibiao Sun ${ }^{2}$ \\ ${ }^{1}$ School of Information Engineering, Dalian University, Dalian 116622, China \\ ${ }^{2}$ School of Control Science and Engineering, Dalian University of Technology, Dalian 116024, China \\ Correspondence should be addressed to Yuan Tian; tianyuan@dlu.edu.cn
}

Received 19 March 2016; Accepted 4 August 2016

Academic Editor: Manuel De la Sen

Copyright (c) 2016 Yuan Tian et al. This is an open access article distributed under the Creative Commons Attribution License, which permits unrestricted use, distribution, and reproduction in any medium, provided the original work is properly cited.

\begin{abstract}
An integrated pest management Gompertz model with interval impulsive control is put forward. Through pest density monitoring, an integrated control strategy is adopted; that is when pest density reaches an environmental damage level, pesticide is used as a control method; when pest density is lower than the damage level, predators as its natural enemy are released in case pest density is higher than the slightly harmful level and predator density is below its maintainable level. The analysis on the existence of order-1 or order-2 periodic orbit is carried out by the construction of Poincaré map of semicontinuous dynamical system. The stability and attractiveness of the periodic state are obtained by geometry approach, which ensures a certain robustness of control. The analytical results presented in the work are validated by numerical simulations for a specific model.
\end{abstract}

\section{Introduction}

Agricultural pests are harmful to the crops, and thus pest management plays an important role in agricultural sustainable development, which also becomes an interesting and significant topic in real life. The traditional and efficient method for pest control is to spray pesticide, which can quickly kill the pest. However, unrestrained use of persistent pesticide not only increases the incidence of pesticide-resistant pest varieties but also inflicts harmful effects on humans through the accumulation of hazardous chemicals in their food chain [1]. An alternative way is the biological control by launching predators or enhancing predators genes to improve the effectiveness of the pest control [2]. This can be achieved by mass production and periodic release of natural enemies of the pest. However, the cultivation of the natural enemy in laboratories is uneasy, and the cost is very high in general. Integrated pest management (IPM) is the comprehensive utilization of agricultural, biological, chemical, and physical methods to control pests with the goal of controlling the number of the pests under an economic threshold (ET) [3-5]. Compared to natural growth process, spraying pesticides (or releasing the natural enemies) can cause the density of pests (or the natural enemies) to change sharply in a short time, which results in the discontinuity of the system states.

In the farmland and forest, the state of pests is always monitored. The administrative authority makes decision (i.e., whether the control should be taken and what kind of control should be adopted) according to the monitored pest level [6]. Based on the adopted control action, a series of integrated pest management models have been built in the literature, for example, the periodic release of predators [7-9]; the periodic release of pests infected by a disease [10]; the periodic release of predators and infected pests [11, 12]; the periodic release of infected pests combined with periodic applications of pesticides [13]; the periodic release of predators and pests combined with periodic applications of pesticides [14-16]; and state-dependent release of predators combined with applications of pesticides [3-5, 17-22].

As far as the state-dependent impulsive control is concerned, the earlier works in pest management belong to Tang et al. [3-5], where the biological and chemical controls are assumed to be taken at the pest economic threshold (ET). Notice that, in real applications, the biological control and chemical control sometimes are adopted at different pest levels; some researchers began to investigate the pest control 
models by assuming that releasing natural enemies and spraying pesticide are taken at different pest thresholds [23-28]. From theoretical and practical points of view, the pest control model with twice impulsive controls looks more reasonable than the model with one impulsive control, and it can be seen as an extension of the one-impulsive-control models.

The ideas of involving biological and chemical controls at different prey densities is interesting and also has practical significance. Biological control with an appropriate yield of release of the predator is adopted in advance, to extend the time for pest density increasing to the damage level (i.e., reduce the operating frequency of adopting chemical control or pesticide). But there exists a problem in modelling the real system; that is, the biological control is adopted when the pest density reaches the first control level, but for a higher pest density between the biological and chemical control levels, there is no control strategy adopted. This is obviously unreasonable. Since the biological control and chemical control are activated at different pest levels, a more reasonable model should also consider the control action when the pest density lies between the two levels; that is to say, when the pest density increases to the chemical level, the chemical control has to be carried out, which causes a certain proportion to pest and predator to be killed. When the pest density increases to or exceeds the biological control activation level but is lower than the chemical control level, the biological control is sufficient, which is also necessary when the predator density is lower than its maintainable level, while in case of low pest density (i.e., below the biological control excitation level) it is not necessity to take any control action. Motivated by this control strategy, the current work presents and studies a preypredator system involving interval state impulsive control.

This paper is organized as follows. In Section 2, a pest control prey-predator model with interval impulsive control is put forward. In Section 3, the Poincaré map, successor function, and some basic definitions are given, followed by a detailed dynamics analysis in case of the chemical control strength. In Section 4, numerical simulations are carried out with a specific model to verify the theoretical results step by step. Finally, conclusions are presented in Section 5.

\section{Model Formulation and Preliminaries}

Let $x(t)$ and $y(t)$ denote the pest and its natural enemy densities at time $t$. Motivated by the control strategy, the following pest management Gompertz model is proposed:

$$
\begin{aligned}
& \frac{d x}{d t}=r x \ln \left(\frac{K}{x}\right)-\beta x y, \\
& \frac{d y}{d t}=y\left(-d+\frac{\lambda \beta x}{1+h x}\right) \\
& x \notin\left[h_{1}, h_{2}\right) \text { or } x \in\left[h_{1}, h_{2}\right), y \geq y_{\tau}(x) \text {, } \\
& \Delta x=0, \\
& \Delta y=\alpha(x), \\
& x \in\left[h_{1}, h_{2}\right), y<y_{\tau}(x), \\
& \Delta x=-p x, \\
& \Delta y=-q y,
\end{aligned}
$$

where $r$ denotes the intrinsic growth rate of the prey in the absence of the predator, $K$ denotes the environment carrying capacity, $\beta$ denotes the predation rate by natural enemies, $\lambda$ denotes the transformation rate and $d$ denotes the death rate of the predator, and $h$ is a positive constant. The control parameters $p$ and $q$ represent the effect of pesticide to prey and predator species. Since the pesticide is relative to the pest, the effect on the predator is limited; here it is assumed that $0<$ $p<1$ and $0<q<q_{\max } \triangleq 1-y_{\tau}\left(h_{1}\right) / \overline{y_{\tau}}\left(h_{2}\right)$. The continuous functions $y_{\tau}(x) \in\left[0, \overline{y_{\tau}}(x)\right]$ and $\alpha_{x}=\alpha(x) \in\left[y_{\tau}(x)-\right.$ $\left.(1-q) y_{\tau}\left(h_{2}\right), \overline{y_{\tau}}(x)-y_{\tau}(x)\right]\left(x \in\left[h_{1}, h_{2}\right)\right)$ are, respectively, the predator reference level and the yield release of the predator, where $\overline{y_{\tau}}(x) \triangleq r \ln (K / x) / \beta$.

The trajectory of the continuous system (1) passing through $P_{0}\left(x_{0}, y_{0}\right)$ is described by $\Gamma(x, y)=\Gamma\left(x_{0}, y_{0}\right)$, where $\Gamma(x, y)$ is the first integral of the continuous system (1). Define the implicit function determined by the low branch above as $y_{\Gamma}^{-1}\left(x,\left(x_{0}, y_{0}\right)\right)\left(\right.$ or $\left.y_{\Gamma}^{-1}\left(x, P_{0}\right)\right)$. Denote

$$
\begin{aligned}
& \Sigma_{1}=\left\{(x, y): x=h_{1}, 0 \leq y \leq y_{\tau}\left(h_{1}\right)\right\}, \\
& \Sigma_{2}=\left\{(x, y): x=h_{2}, 0 \leq y \leq \overline{y_{\tau}}\left(h_{2}\right)\right\}, \\
& \Sigma_{3}= \begin{cases}\left\{(x, y) \mid x=(1-p) h_{2}, 0 \leq y \leq y_{\tau}\left((1-p) h_{2}\right)\right\} & \text { if } 0<h_{1} \leq(1-p) h_{2}, \\
\varnothing & \text { if } 0<(1-p) h_{2} \leq h_{1},\end{cases} \\
& \Sigma_{1}^{+}=\left\{(x, y): x=h_{1}, y_{h_{1}}<y \leq y_{\tau}\left(h_{1}\right)+\alpha_{h_{1}}\right\}, \\
& \Sigma_{2}^{+}=\emptyset, \\
& \Sigma_{3}^{+}= \begin{cases}\left\{(x, y) \mid x=(1-p) h_{2}, y_{(1-p) h_{2}}<y \leq y_{\tau}\left((1-p) h_{2}\right)+\alpha_{(1-p) h_{2}}\right\} & \text { if } 0<h_{1} \leq(1-p) h_{2}, \\
\left\{(x, y) \mid x=(1-p) h_{2}, 0<y \leq(1-p) \overline{y_{\tau}}\left(h_{2}\right)\right\} & \text { if } 0<(1-p) h_{2} \leq h_{1} .\end{cases}
\end{aligned}
$$


Definition 1 (Poincaré map). For any trajectory $\mathrm{O}^{+}\left(P_{n}^{+}, t_{n}\right)$ in system (1) starting from point $P_{n}^{+}\left((1-p) h_{2}, y_{n}^{+}\right)$on $\Sigma_{3}^{+}$, there will be two cases: (1) for $0<h_{1} \leq(1-p) h_{2}$, the trajectory first reaches the point $P_{n+1}^{-}\left(h_{2}, y_{n+1}^{-}\right)$on section $\Sigma_{2}$; then, it jumps from $P_{n+1}^{-}$to the point $P_{n+1}\left((1-p) h_{2},(1-q) y_{n+1}^{-}\right)$on $\Sigma_{3}$ due to the impulsive effects $\Delta x=(1-p) x$ and $\Delta y=(1-q) y$ and then jumps from $P_{n+1}$ to $P_{n+1}^{+}\left((1-p) h_{2}, y_{n+1}^{+}\right)$on $\Sigma_{3}^{+}$due to the impulsive effects $\Delta x=0$ and $\Delta y=\alpha_{(1-p) h_{2}}$; (2) for $0<(1-$ p) $h_{2}<h_{1}$, the trajectory first reaches the point $P_{n+1}\left(h_{1}, y_{n+1}\right)$ on section $\Sigma_{1}$; then, it jumps from $P_{n+1}$ to $P_{n+1}^{\prime}\left(h_{1}, y_{n+1}+\alpha_{h_{1}}\right)$ on $\Sigma_{1}^{+}$due to the impulsive effects $\Delta x=0$ and $\Delta y=\alpha_{h_{1}}$ and then the trajectory starting from $P_{n+1}^{\prime}$ reaches the point $P_{n+1}^{-}\left(h_{2}, y_{n+1}^{-}\right)$on section $\Sigma_{2}$ then jumps from $P_{n+1}^{-}$to $P_{n+1}^{+}((1-$ p) $\left.h_{2},(1-q) y_{n+1}^{-}\right)$on $\Sigma_{3}^{+}$due to the impulsive effects $\Delta x=$ $(1-p) x$ and $\Delta y=(1-q) y$. In any case, $y_{n+1}^{+}$is a function of $y_{n}^{+}, p, q, \alpha_{h_{1}}$, and $\alpha_{(1-p) h_{2}}$. The Poincaré map $\mathscr{P}$ on $\Sigma_{3}^{+}$is defined as

$$
\mathscr{P}\left(y_{n}^{+}\right)=y_{n+1}^{+}
$$

Definition 2 (successor function). The successor function on $\Sigma_{3}^{+}$is defined by

$$
s\left(P_{n}^{+}\right) \triangleq y_{P_{n+1}^{+}}-y_{P_{n}^{+}}=\mathscr{P}\left(y_{P_{n}^{+}}\right)-y_{P_{n}^{+}},
$$

which is continuous on $\Sigma_{3}^{+}$, where $\mathscr{P}(y)$ is the Poincaré map determined by (3).

Definition 3 (periodic solution). The solution of system (1) passing through $P \in \Sigma_{3}^{+}$is called periodic if $s(P)=0$.

\section{Dynamic Analysis of System (1)}

As illustrated in Figure 1, system (1) has three equilibria $O(0,0), R(K, 0)$, and $E\left(x^{*}, y^{*}\right)$, where $x^{*}=d /(\lambda \beta-d h)$ and $y^{*}=r \ln (K(\lambda \beta-d h) / d) / \beta$ if $\left(H_{1}\right): K>\bar{K} \triangleq d /(\lambda \beta-d h)$ and $\lambda \beta>d h$ holds. The reference level $y_{\tau}(x)$ is established by a statistics method in practice, which reflects a certain consistency of the system; that means the biological control taken at any level should take the same or similar effect in determining the system's dynamics. Thus, in this study a trajectory of the solution of system (1) starting from the point $T_{0}$ on $x=h_{1}$ is selected as a predator reference. To make practical sense, the study is restricted in the left region of $E\left(x^{*}, y^{*}\right)$; that is, $0<h_{1}<h_{2}<x^{*}$.

3.1. Existence of Periodic Solution. The discussions will be divided into three cases according to the magnitude between $h_{1}$ and $(1-p) h_{2}$ : the position of the trajectory $\widehat{T_{0} T_{1}}$, the magnitude of $\alpha$ and $q$, and the location of the point $P_{n}^{+}$.

Case $1\left((1-p) h_{2}\right.$ is greater than or equal to $\left.h_{1}\right)$. Suppose the intersection point between the line $x=(1-p) h_{2}$ and the trajectory staring from $T_{0}$ is $T_{2}$. The trajectory starting from the point $P_{n}^{+}$between $T_{2}$ and $M_{2}$ intersects with $x=h_{2}$ at $P_{n+1}^{-}$and then jumps to the point $P_{n+1}$ on $x=(1-p) h_{2}$ under the effect of pulse. If the point $P_{n+1} \in \Sigma_{3}^{+}$, then it will jump upward to the point $P_{n+1}^{+}$.

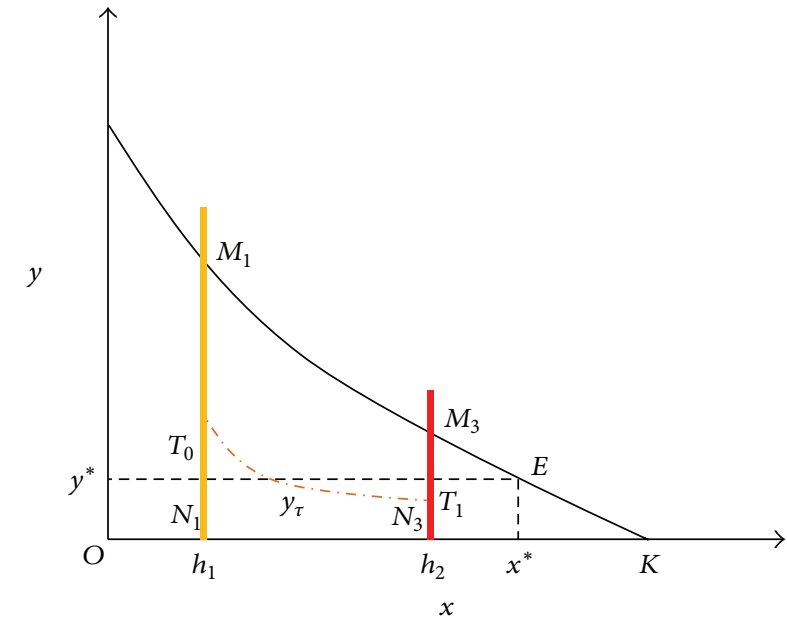

FIgURE 1: The system structural diagram.

Case $1.1\left(P_{n+1}^{+}\right.$overlaps with $\left.P_{n}^{+}\right)$. Then, the curve $\widehat{P_{n}^{+} P_{n+1}^{-}} P_{n+1} P_{n+1}^{+}$constitutes an order-1 periodic orbit (see Figure 2(a)).

Case $1.2\left(P_{n+1}^{+}\right.$is under $\left.P_{n}^{+}\right)$. Then, there is $s\left(P_{n}^{+}\right)<0$. Next, choose a point $Q_{n}^{+} \in \Sigma_{3}^{-}$adjacent to $T_{2}$ sufficiently (i.e., $\left|T_{2} Q_{n}^{+}\right|<\varepsilon$ ). The trajectory of system (1) starting from $Q_{n}^{+}$ intersects with $x=h_{2}$ at $Q_{n+1}^{-}$then jumps to $Q_{n+1} \in \Sigma_{3}^{+}$under impulsive effects $\Delta x=-p x$ and $\Delta y=-q y$ and next jumps to $Q_{n+1}^{+}$above $Q_{n}^{+}$under impulsive effects $\Delta x=0$ and $\Delta y=$ $\alpha_{(1-p) h_{2}}$. Thus, there is $s\left(Q_{n}^{+}\right)>0$ (see Figure 2(b)), which means that an order-1 periodic solution exists.

Case $1.3\left(P_{n+1}^{+}\right.$is above $\left.P_{n}^{+}\right)$. In this case, there is $s\left(P_{n}^{+}\right)>0$. Then, choose a point $Q_{n}^{+}$between $M_{2}$ and $P_{n+1}^{+}$, which is close to $M_{2}$ sufficiently; the trajectory starting from $Q_{n}^{+}$intersects with $x=h_{2}$ at $Q_{n+1}^{-}$and then jumps to $Q_{n+1}$ on $x=(1-$ p) $h_{2}$ under impulsive effects $\Delta x=-p x$ and $\Delta y=-q y$. If $Q_{n+1} \in \Sigma_{p}^{+}$, then it jumps to $Q_{n+1}^{+} \in \Sigma_{3}^{-}$under impulsive effects $\Delta x=0$ and $\Delta y=\alpha_{(1-p) h_{2}}$, and $Q_{n+1}^{+}$must be under $Q_{n}^{+}$; then, there is $s\left(Q_{n}^{+}\right)<0$ (see Figure 2(c)); that is, an order-1 periodic solution exists. Otherwise, let $R^{+}$be the point on $x=(1-p) h_{2}$ such that $\mathscr{P}\left(y_{R}\right)=y_{T_{2}}+\alpha_{(1-p) h_{2}}$. If $s(R) \leq 0$, then an order-1 periodic solution exists.

Case $2\left((1-p) h_{2}\right.$ is smaller than $\left.h_{1}\right)$. Suppose the trajectory starting from $P_{n}^{+} \in \Sigma_{3}$ intersects with the line $x=h_{1}$ at $P_{n+1} \in$ $\Sigma_{1}^{+}$; then, it jumps to the point $P_{n+1}^{\prime} \in \Sigma_{1}^{-}$under the impulsive effects $\Delta x=0$ and $\Delta y=\alpha_{h_{1}}$. And then the trajectory starting from $P_{n+1}^{\prime}$ intersects with $x=h_{2}$ at point $P_{n+1}^{-}$and then jumps to $\Sigma_{3}$ at the point $P_{n+1}^{+}$under the effect of pulses $\Delta x=-p x$ and $\Delta y=-q y$. According to the relative position of $P_{n+1}^{+}$and $P_{n}^{+}$, there are three cases to be considered.

Case $2.1\left(P_{n+1}^{+}\right.$overlaps with $\left.P_{n}^{+}\right)$. Then, the curve $\widehat{P_{n}^{+} P_{n+1}} \widehat{P_{n+1}^{\prime} P_{n+1}^{+}}$constitutes an order-2 periodic orbit (see Figure 3(a)) 


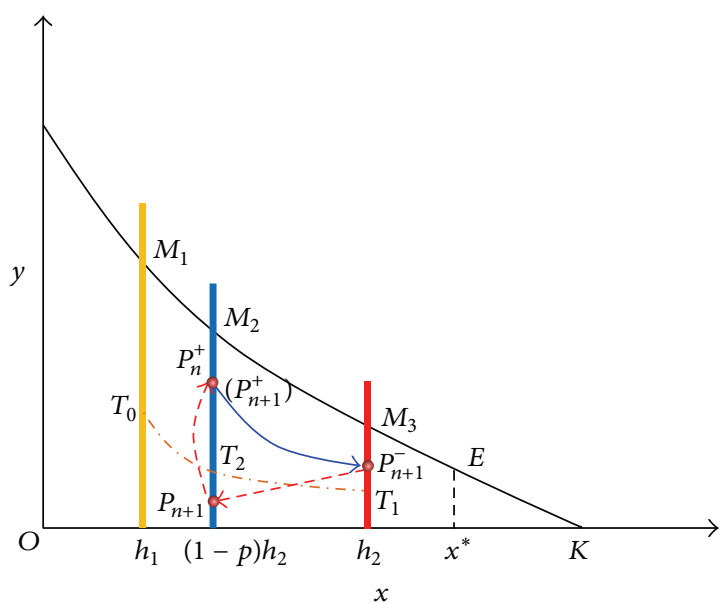

(a)

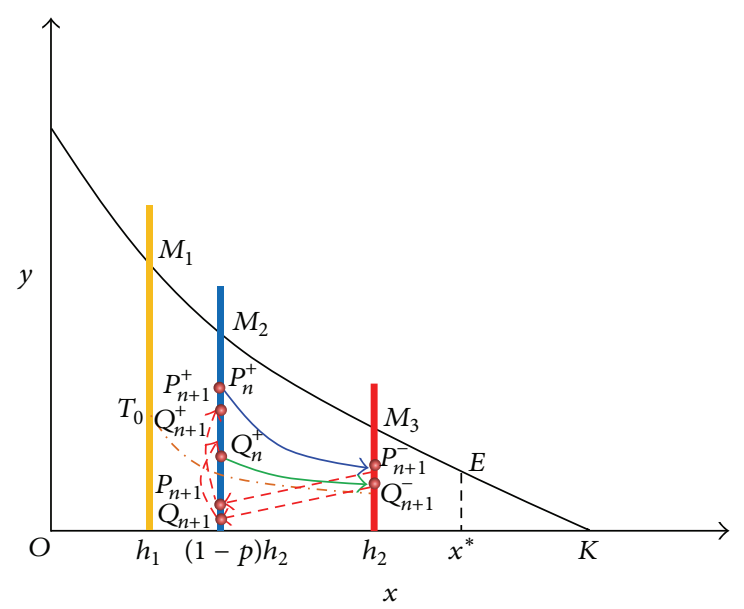

(b)

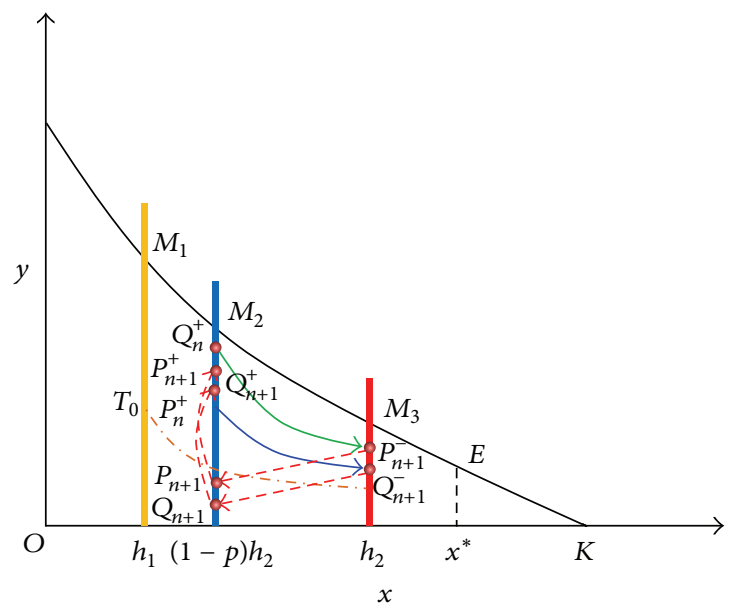

(c)

Figure 2: The trajectory of system (1) when $(1-p) h_{2}$ is greater than $h_{1}$. (a) $P_{n+1}^{+}$overlaps with $P_{n}^{+}$; (b) $P_{n+1}^{+}$is under $P_{n}^{+}$; (c) $P_{n+1}^{+}$is above $P_{n}^{+}$.

Case $2.2\left(P_{n+1}^{+}\right.$is under $\left.P_{n}^{+}\right)$. Then, there is $s\left(P_{n}^{+}\right)<0$. Next, choose a point $Q_{n}^{+} \in \Sigma_{3}$ adjacent to $N_{2}$ (i.e., $\left|Q_{n}^{+} N_{2}\right|<\varepsilon$ ), where $N_{2}$ is the phase point of $T_{1}$. The trajectory of system (1) starting from $Q_{n}^{+}$intersects with $x=h_{1}$ at point $Q_{n+1}$ and then jumps to the point $Q_{n+1}^{\prime} \in \Sigma_{1}^{-}$under the impulsive effects $\Delta x=0$ and $\Delta y=\alpha_{h_{1}}$. The trajectory starting from $Q_{n+1}^{\prime}$ intersects with $x=h_{2}$ at point $Q_{n+1}^{-}$and then jumps to a point $Q_{n+1}^{+} \in \Sigma_{3}$ under the impulsive effects $\Delta x=-p x$ and $\Delta y=$ $-q y$. Since $Q_{n}^{+}$is close to $N_{2}$, then $Q_{n+1}^{+}$must be above $Q_{n}^{+}$; then, there is $s\left(Q_{n}^{+}\right)>0$ (see Figure $3(\mathrm{~b})$ ), which means that an order-2 periodic solution exists.

Case $2.3\left(P_{n+1}^{+}\right.$is above $\left.P_{n}^{+}\right)$. In this case, there is $s\left(P_{n}^{+}\right)>0$. Let $S_{0}$ be a point on $x=h_{1}$ such that the trajectory starting from $S_{0}$ intersects with the line $x=h_{1}$ at $T_{0}$. If the trajectory starting from $S_{0}$ does not intersect with $\Sigma_{3}$, then choose the point $S\left((1-p) h_{2},(1-q) \overline{y_{\tau}}\left(h_{2}\right)\right) \in \Sigma_{3}$ as $Q_{n}^{+}$. Obviously there is $s\left(Q_{n}^{+}\right)<0$. If the trajectory starting from $S_{0}$ intersects with $\Sigma_{3}$ at $S_{1}$ and $S_{2}$ with $y_{S_{1}} \geq y_{S_{2}}$, then choose the point from $S_{2}$ and $S$ with smaller $y$ label as $Q_{n}^{+}$. The trajectory starting from
$Q_{n}^{+}$intersects with $x=h_{1}$ at $Q_{n+1}$ below $T_{0}$ and then jumps to $Q_{n+1}^{\prime} \in \Sigma_{1}^{-}$. The trajectory starting from $Q_{n+1}^{\prime}$ intersects with $x=h_{2}$ at $Q_{n+1}^{-}$and then jumps to $Q_{n+1}^{+} \in \Sigma_{3}$. If $\mathscr{P}\left(y_{Q_{n}^{+}}\right) \leq$ $y_{Q_{n}^{+}}$, there is $s\left(Q_{n}^{+}\right)<0$ (see Figure 3(c)), which means that an order-2 periodic solution exists.

\subsection{Stability of the Periodic Solutions}

Theorem 4. For the case $0<h_{1} \leq(1-p) h_{2}$, if the order-1 periodic solution exists, it is unique and orbitally asymptotically stable.

Proof.

(1) The Uniqueness of the Order-1 Periodic Solution. Assume system (1) admits two different order-1 periodic solutions $P_{n}^{+} \widehat{P_{n+1}^{-} P_{n+1}} P_{n}^{+}$and $Q_{n}^{+} \widehat{Q_{n+1}^{-} Q_{n+1}} Q_{n}^{+}$which start from $P_{n}^{+}$and $Q_{n}^{+}$on $x=(1-p) h_{2}$ with $y_{P_{n}^{+}}>y_{Q_{n}^{+}}$, respectively. Then, there is $s\left(P_{n}^{+}\right)=s\left(Q_{n}^{+}\right)=0$. Denote $d_{P_{n}^{+} Q_{n}^{+}}(x)=y_{P_{n}^{+}}(x)-y_{Q_{n}^{+}}(x)$, 


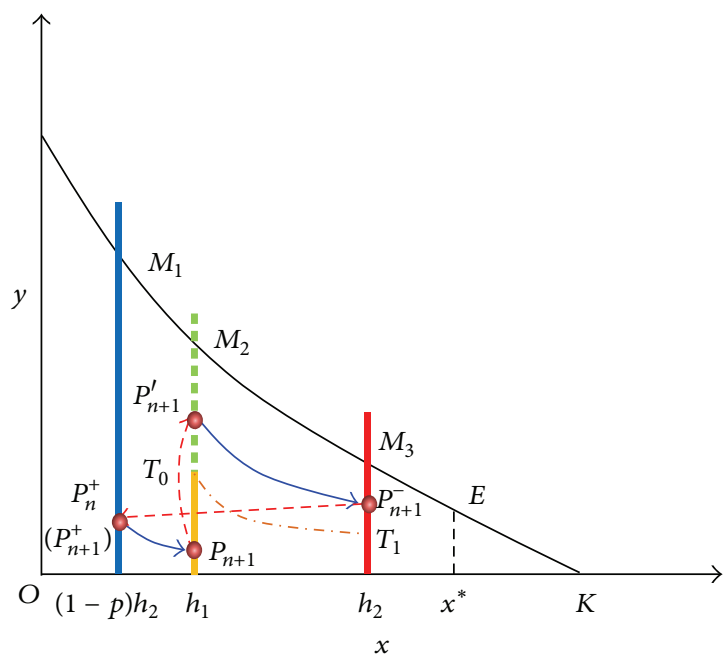

(a)

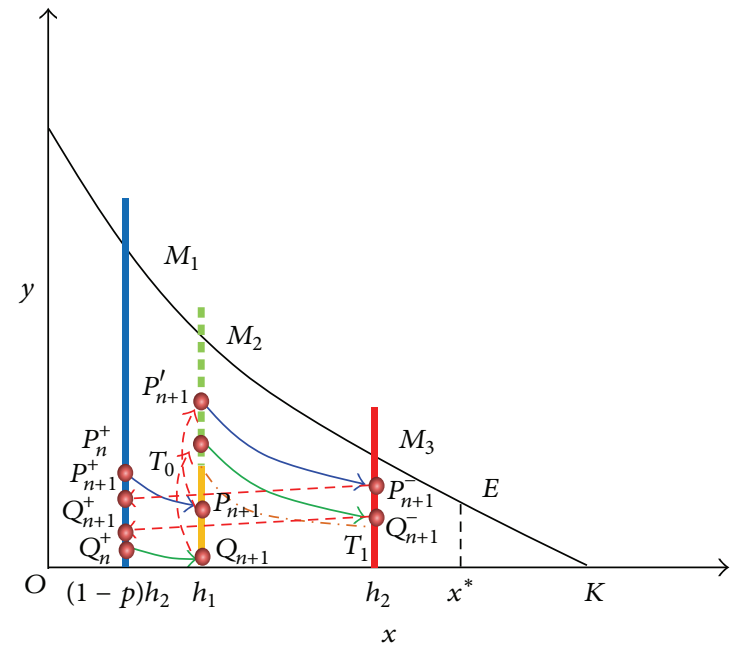

(b)

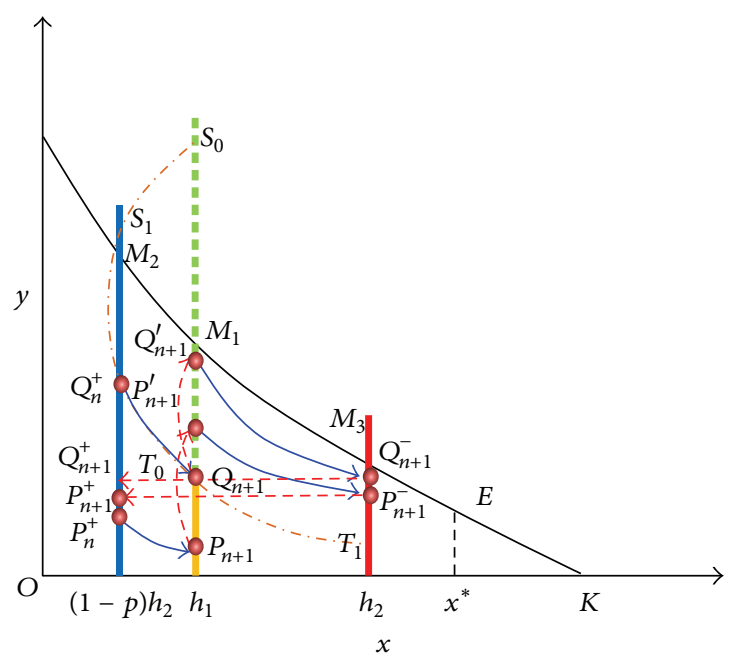

(c)

FIGURE 3: The trajectory of system (1) when $(1-p) h_{2}$ is less than $h_{1}$. (a) $P_{n+1}^{+}$overlaps with $P_{n}^{+}$; (b) $P_{n+1}^{+}$is under $P_{n}^{+}$; (c) $P_{n+1}^{+}$is above $P_{n}^{+}$.

where $y_{P_{n}^{+}}(x)=y_{\Gamma}^{-1}\left(x, P_{n}^{+}\right)$and $y_{Q_{n}^{+}}(x)=y_{\Gamma}^{-1}\left(x, Q_{n}^{+}\right)$with $x \in\left[h_{1}, h_{2}\right]$. Then, there is

$$
\begin{aligned}
d_{P_{n}^{+} Q_{n}^{+}}^{\prime}(x) & =y_{P_{n}^{+}}^{\prime}(x)-y_{Q_{n}^{+}}^{\prime}(x) \\
& =\frac{\gamma \beta x-d}{x} \frac{r \ln (K / x)}{(r \ln (K / x)-\beta \eta)^{2}}\left(y_{P_{n}^{+}}-y_{Q_{n}^{+}}\right),
\end{aligned}
$$

which means that $d_{P_{n}^{+} Q_{n}^{+}}^{\prime}(x)<0$; that is, $d_{P_{n}^{+} Q_{n}^{+}}(x)$ is a monotone decreasing function on $\left[h_{1}, h_{2}\right]$. Then, it yields that

$$
d_{P_{n}^{+} Q_{n}^{+}}\left(h_{1}\right)>d_{P_{n}^{+} Q_{n}^{+}}\left(h_{2}\right)>(1-q) d_{P_{n}^{+} Q_{n}^{+}}\left(h_{2}\right) ;
$$

that is, $d\left(P_{n}^{+}, Q_{n}^{+}\right)>d\left(P_{n+1}^{-}, Q_{n+1}^{-}\right)>d\left(P_{n+1}, Q_{n+1}\right)$. Then, there is

$$
\begin{aligned}
\alpha_{(1-p) h_{2}} & =d\left(P_{n}^{+}, Q_{n}^{+}\right)+d\left(Q_{n}^{+}, P_{n+1}\right) \\
& >d\left(P_{n+1}, Q_{n+1}\right)+d\left(Q_{n}^{+}, P_{n+1}\right)=\alpha_{(1-p) h_{2}}
\end{aligned}
$$

which leads to a contradiction. Thus, the order-one periodic solution is unique.

(2) Stability of the Order-1 Periodic Solution. Suppose the impulsive point of $T_{2}$ is $T_{2}^{+}$; obviously, the trajectory of system (1) starting from any point will arrive at the segment $\overline{T_{2} T_{2}^{+}}$. Thus, it is only necessary to consider the tendency of the trajectory starting from $\overline{T_{2} T_{2}^{+}}$. If $y_{P_{n}^{+}}<y_{T_{2}^{+}}$, we should consider the tendency of the trajectory starting from $\overline{T_{2} P_{n}^{+}}$and $\overline{P_{n}^{+} T_{2}^{+}}$, respectively. For any $M_{0}^{+} \in \overline{T_{2} P_{n}^{+}}$, a sequence $\left.\left\{M_{k}^{+}\right\}\right|_{k=0,1,2, \ldots}$ on $\overline{T_{2} P_{n}^{+}}$can be obtained such that $y_{M_{k+1}^{+}}=y_{M_{k}^{+}}+s\left(M_{k}^{+}\right)$; and for any $N_{0}^{+} \in \overline{P_{n}^{+} T_{2}^{+}}$, a sequence $\left.\left\{N_{k}^{+}\right\}\right|_{k=0,1,2, \ldots}$ on $\overline{P_{n}^{+} T_{2}^{+}}$can be obtained such that $y_{N_{k+1}^{+}}=$ $y_{N_{k}^{+}}+s\left(N_{k}^{+}\right)$. From the proof of the uniqueness of the orderone periodic, it can be observed that the successor function $s$ satisfies the following properties:

(i) $s\left(M_{0}^{+}\right)=s\left(N_{0}^{+}\right)=0$ if and only if $M_{0}^{+}=N_{0}^{+}=P_{n}^{+}$; 
(ii) $s\left(M_{0}^{+}\right)>0$ for $M_{0}^{+} \in \overline{T_{2} P_{n}^{+}}, M_{0}^{+} \neq P_{n}^{+}$;

(iii) $s\left(N_{0}^{+}\right)<0$ for $N_{0}^{+} \in \overline{P_{n}^{+} T_{2}^{+}}, N_{0}^{+} \neq P_{n}^{+}$.

This means that $\left\{y_{M_{k}^{+}}\right\}$is a monotone increasing sequence with an upper bound $y_{P_{n}^{+}}$, and $\left\{y_{N_{k}^{+}}\right\}$is a monotone decreasing sequence with a lower bound $y_{P_{n}^{+}}$; then, there exist $y_{M_{1}^{+}}$and $y_{N_{1}^{+}}$such that $y_{M_{k}^{+}} \rightarrow y_{M_{1}^{+}}$and $\left\{y_{N_{k}^{+}}\right\} \rightarrow y_{N_{1}^{+}}$when $k \rightarrow \infty$. But $s\left(M_{1}^{+}\right)=s\left(N_{1}^{+}\right)=0$ implies that $M_{1}^{+}=N_{1}^{+}=P^{+}$. Thus, the unique order-1 periodic ${P_{n}^{+}}_{P_{n+1}^{-} P_{n+1}} P_{n}^{+}$is orbitally asymptotically stable. Since the points $M_{0}^{+}$and $N_{0}^{+}$are arbitrary, the orbit asymptotical stability implies the global attractiveness.

Theorem 5. For the case $0<(1-p) h_{2}<h_{1}$, if the order2 periodic solution exists, it is unique, orbitally asymptotically stable, and globally attractive.

Proof.

(1) The Uniqueness of the Order-2 Periodic Solution. Assume system (1) admits two different period-2 solutions $P_{n}^{+} P_{n+1} \widehat{P_{n+1}^{\prime} P_{n+1}^{-}} P_{n}^{+}$and $Q_{n}^{+} Q_{n+1} \widehat{Q_{n+1}^{\prime}} Q_{n+1}^{-} Q_{n}^{+}$which start from $P_{n}^{+}$and $Q_{n}^{+}$with $y_{P_{n}^{+}}>y_{Q_{n}^{+}}$, respectively. Then, there is $s\left(P_{n}^{+}\right)=s\left(Q_{n}^{+}\right)=0$ and

$$
\begin{aligned}
d\left(P_{n}^{+}, Q_{n}^{+}\right) & >d\left(P_{n+1}, Q_{n+1}\right)>d\left(P_{n+1}^{-}, Q_{n+1}^{-}\right) \\
& >d\left(P_{n}^{+}, Q_{n}^{+}\right)
\end{aligned}
$$

which leads to a contradiction. Thus, the order- 2 periodic solution is unique.

(2) The Stability of the Order-2 Periodic Solution. Assume that $T_{1}^{+}$and $M_{3}^{+}$are the impulsive points of $T_{1}$ and $M_{3}$ under the pulses $\Delta x=(1-p) x$ and $\Delta y=(1-q) y$, respectively. The trajectory $T_{0} T_{1}$ meets with $x=(1-p) h_{2}$ at the point $T_{2}$ in the opposite direction; $T_{0}^{\prime}$ is the impulsive point of $T_{0}$. Obviously, the trajectory of system (1) starting from any point will arrive at the segment $\overline{T_{1}^{+} M_{3}^{+}}$if $y_{M_{3}^{+}}<y_{T_{2}}$ or $\overline{T_{1}^{+} T_{2}}$ if $y_{M_{3}^{+}}>y_{T_{2}}$. Thus, it is only necessary to consider the tendency of the trajectory starting from $\overline{T_{1}^{+} M_{3}^{+}}$or $\overline{T_{1}^{+} T_{2}}$. Denote the order2 periodic by $P_{n}^{+} P_{n+1} \widehat{P_{n+1}^{\prime} P_{n+1}^{-}} P_{n}^{+}$with $P_{n}^{+}$on the segment $\overline{T_{1}^{+} M_{3}^{+}}$or $\overline{T_{1}^{+} T_{2}}$. Similarly, let us consider the tendency of the trajectory starting from $\overline{P_{n}^{+} M_{3}^{+}}$or $\overline{P_{n}^{+} T_{2}}$ and $\overline{T_{1}^{+} P_{n}^{+}}$, respectively. For any $Q_{0}^{+} \in \overline{P_{n}^{+} M_{3}^{+}}$or $Q_{0}^{+} \in \overline{P_{n}^{+} T_{2}}$, a sequence $\left.\left\{Q_{k}^{+}\right\}\right|_{k=0,1,2, \ldots}$ can be obtained such that $y_{Q_{k+1}^{+}}=y_{Q_{k}^{+}}+s\left(Q_{k}^{+}\right)$; and for any $R_{0}^{+} \in \overline{T_{1}^{+} P_{n}^{+}}$, a sequence $\left.\left\{R_{k}^{+}\right\}\right|_{k=0,1,2, \ldots}$ on $\overline{T_{1}^{+} P_{n}^{+}}$can be obtained such that $y_{R_{k+1}^{+}}=y_{R_{k}^{+}}+s\left(R_{k}^{+}\right)$. From the proof of the uniqueness of the order- 2 periodic solution, it can be observed that the successor function $s$ satisfies the following properties:

(i) $s\left(Q_{0}^{+}\right)=s\left(R_{0}^{+}\right)=0$ if and only if $Q_{0}^{+}=R_{0}^{+}=P_{n}^{+}$; (ii) $s\left(Q_{0}^{+}\right)>0$ for $Q_{0}^{+} \in \overline{P_{n}^{+} M_{3}^{+}}, Q_{0}^{+} \neq P_{n}^{+}$or $Q_{0}^{+} \in \overline{P_{n}^{+} T_{2}}$, $Q_{0}^{+} \neq P_{n}^{+}$;

(iii) $s\left(R_{0}^{+}\right)<0$ for $R_{0}^{+} \in \overline{T_{1}^{+} P_{n}^{+}}, R_{0}^{+} \neq P_{n}^{+}$.

This means that $\left\{y_{\mathrm{Q}_{k}^{+}}\right\}$is a monotone increasing sequence with an upper bound $y_{P_{n}^{+}}$, and $\left\{y_{R_{k}^{+}}\right\}$is a monotone decreasing sequence with a lower bound $y_{P_{n}^{+}}$; then, there exist $y_{Q^{+}}$and $y_{R^{+}}$such that $y_{Q_{k}^{+}} \rightarrow y_{Q^{+}}$and $\left\{y_{R_{k}^{+}}\right\} \rightarrow y_{R^{+}}$when $k \rightarrow \infty$. But $s\left(Q^{+}\right)=s\left(R^{+}\right)=0$ implies that $Q^{+}=R^{+}=P_{n}^{+}$. Thus, the unique order-2 periodic solution $P_{n}^{+} P_{n+1} \widehat{P_{n+1}^{\prime} P_{n+1}^{-}} P_{n}^{+}$is orbitally asymptotically stable. Since the points $Q_{0}^{+}$and $R_{0}^{+}$are arbitrary, the orbit asymptotical stability implies the global attractiveness.

\section{Numerical Simulations}

In this section, a specific example is given to verify the theoretical results obtained in the previous section by considering the change of the control parameters $p, q$, and $\alpha_{x}$. Let $r=1.2$, $K=100, \beta=0.5, \lambda=0.16, d=0.4$, and $h=0.18$. With a simple calculation, the steady state of free system is $E\left(x^{*}, y^{*}\right)=E(50,1.664)$. The chemical control is assumed to be taken at $h_{2}=40$, and the biological control is assumed to be taken at $h_{1}=20$. The predator reference level at $h_{1}$ is assumed to be about $10 \%$ of steady predator density of the free system; that is, $y_{\tau}\left(h_{1}\right)=0.1664$. The curve $r \ln (K / x)-\beta y=0$ intersects with $x=h_{1}$ and $x=h_{2}$ at the points $(20,3.8627)$ and $(40,2.1991)$, respectively. Then, there is $\overline{y_{\tau}}\left(h_{1}\right)=3.8627$ and $\overline{y_{\tau}}\left(h_{2}\right)=2.1991$.

4.1. Verification for the Case $0<h_{1} \leq(1-p) h_{2}$. Let $p=$ $q=0.4$. Then, there is $(1-p) h_{2}=24$. The predator reference level at $(1-p) h_{2}$ is about $y_{\tau}\left((1-p) h_{2}\right)=0.1651$. The curve $r \ln (K / x)-\beta y=0$ intersects with $x=(1-p) h_{2}$ at the point $(24,3.4251)$; then, there is $\overline{y_{\tau}}\left((1-p) h_{2}\right)=3.4251$. For the yield release $\alpha_{(1-p) h_{2}}=0.1$, an order-1 periodic solution is formed, as illustrated in Figure 4.

When $\alpha_{(1-p) h_{2}}$ increases to 0.2 , that is, $\alpha_{(1-p) h_{2}}=0.2$, the order-1 periodic solution disappears, and an order- 2 periodic solution is formed, as illustrated in Figure 5.

For the yield release $\alpha_{(1-p) h_{2}}=0.5$, an order-3 periodic solution is formed (Figure 6).

4.2. Verification for the Case $0<(1-p) h_{2}<h_{1}$. Let $p=0.6$ and $q=0.2$. Then, $(1-p) h_{2}=16$. For the release $\alpha_{h_{1}}=0.04$, an order-2 periodic solution is formed (Figure 7).

For the yield release $\alpha_{h_{1}}=0.1$, an order-3 periodic solution is formed (Figure 8).

For the yield release $\alpha_{h_{1}}=0.15$, an order- 4 periodic solution is formed (Figure 9).

For the yield release $\alpha_{h_{1}}=0.25$, an order-5 periodic solution is formed (Figure 10).

\section{Conclusion}

In this work, a pest control prey-predator model was analyzed, where the biological control and chemical control are 


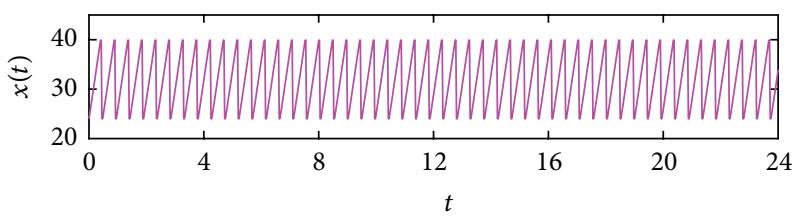

(a)

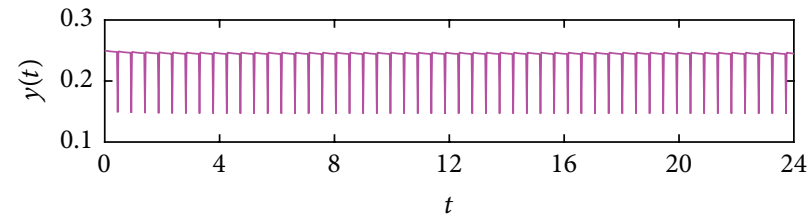

(b)

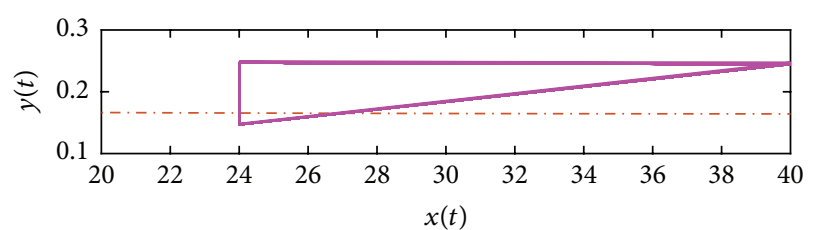

(c)

FIGURE 4: The change in prey density (a), predator density (b), and phase diagrams (c) starting from $\left(x_{0}, y_{0}\right)=(24,0.25)$ with $p=0.4, q=0.4$, $h_{1}=20, h_{2}=40, y_{\tau}\left(h_{1}\right)=0.1664, y_{\tau}\left((1-p) h_{2}\right)=0.1651$, and $\alpha_{(1-p) h_{2}}=0.1$.

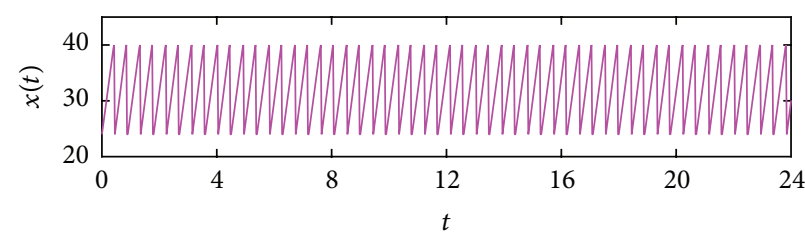

(a)

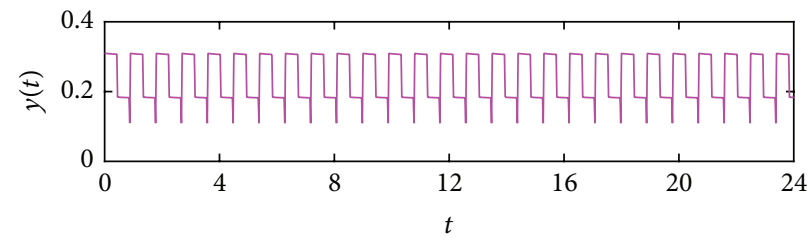

(b)

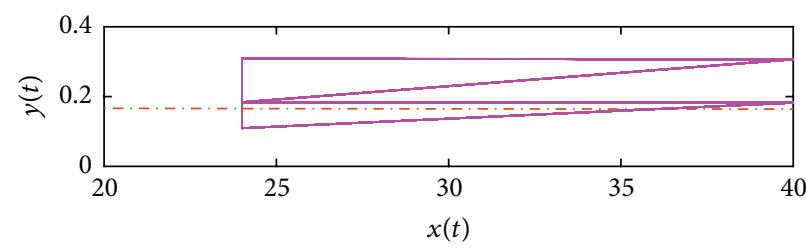

(c)

FIGURE 5: The change in prey density (a), predator density (b), and phase diagrams (c) starting from $\left(x_{0}, y_{0}\right)=(24,0.25)$ with $p=0.4, q=0.4$, $h_{1}=20, h_{2}=40, y_{\tau}\left(h_{1}\right)=0.1664, y_{\tau}\left((1-p) h_{2}\right)=0.1651$, and $\alpha_{(1-p) h_{2}}=0.2$.

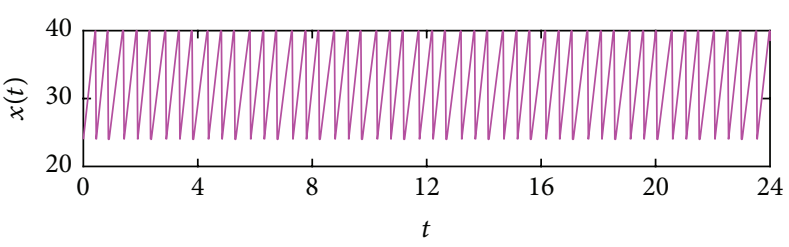

(a)

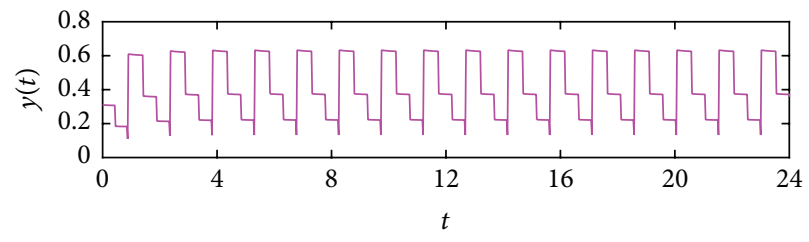

(b)

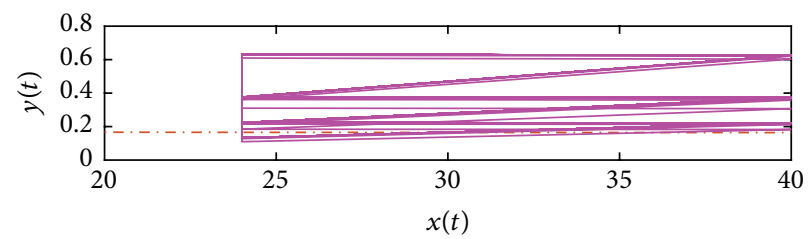

(c)

FIGURE 6: The change in prey density (a), predator density (b), and phase diagrams (c) starting from $\left(x_{0}, y_{0}\right)=(24,0.25)$ with $p=0.4, q=0.4$, $h_{1}=20, h_{2}=40, y_{\tau}\left(h_{1}\right)=0.1664, y_{\tau}\left((1-p) h_{2}\right)=0.1651$, and $\alpha_{(1-p) h_{2}}=0.5$. 


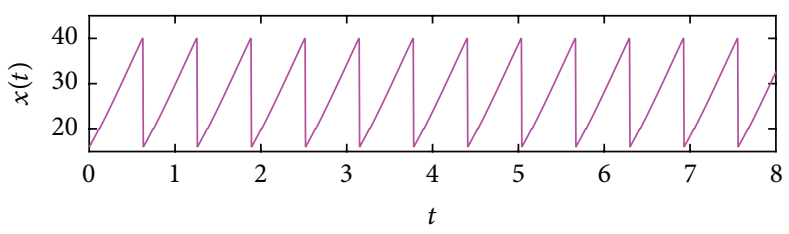

(a)

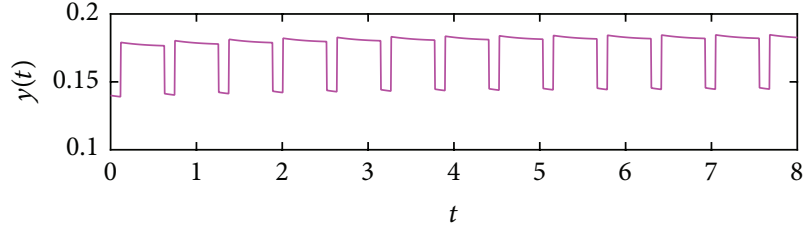

(b)

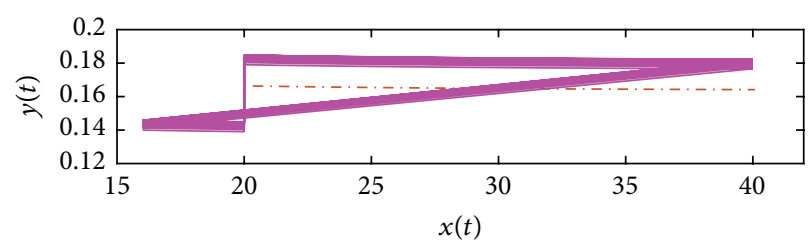

(c)

FIGURE 7: The change in prey density (a), predator density (b), and phase diagrams (c) starting from $\left(x_{0}, y_{0}\right)=(16,0.14)$ with $p=0.6, q=0.2$, $h_{1}=20, h_{2}=40, y_{\tau}\left(h_{1}\right)=0.1664$, and $\alpha_{h_{1}}=0.04$.

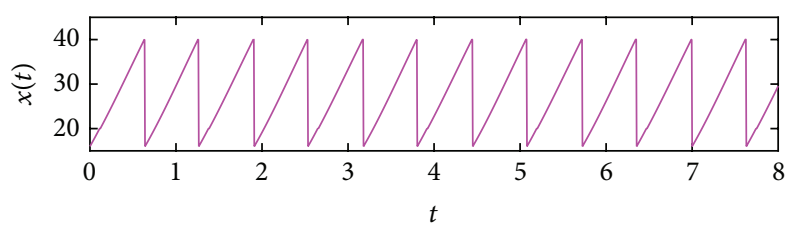

(a)

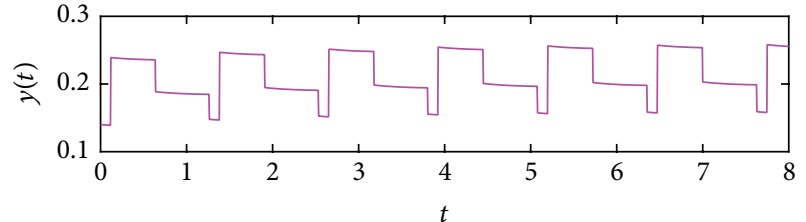

(b)

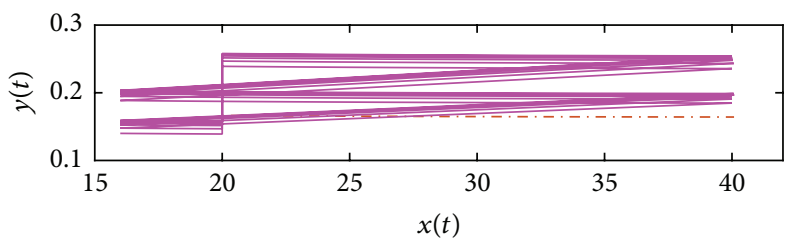

(c)

Figure 8: The change in prey density (a), predator density (b), and phase diagrams (c) starting from $\left(x_{0}, y_{0}\right)=(16,0.14)$ with $p=0.6, q=0.2$, $h_{1}=20, h_{2}=40, y_{\tau}\left(h_{1}\right)=0.1664$, and $\alpha_{h_{1}}=0.1$.

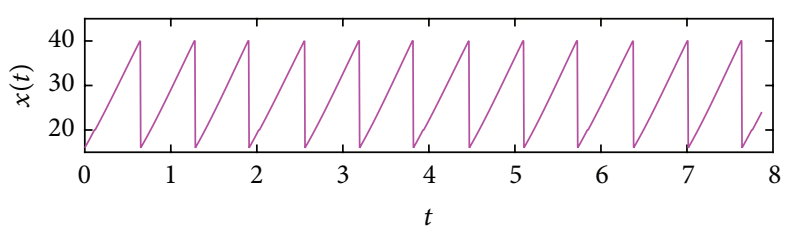

(a)

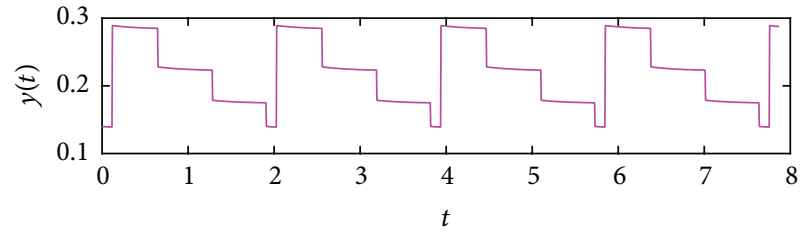

(b)

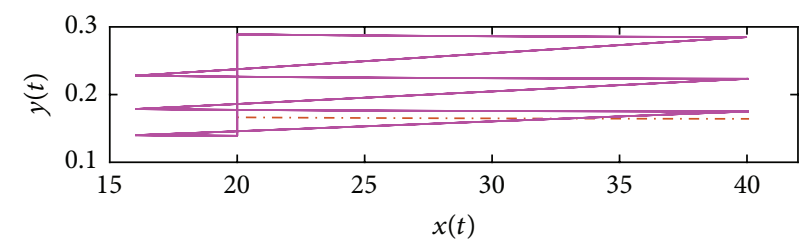

(c)

FIGURE 9: The change in prey density (a), predator density (b), and phase diagrams (c) starting from $\left(x_{0}, y_{0}\right)=(16,0.14)$ with $p=0.6, q=0.2$, $h_{1}=20, h_{2}=40, y_{\tau}\left(h_{1}\right)=0.1664$, and $\alpha_{h_{1}}=0.15$. 


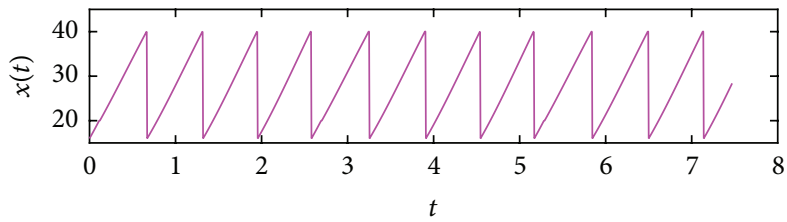

(a)

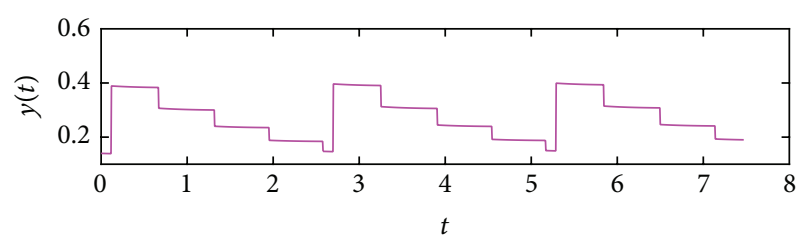

(b)

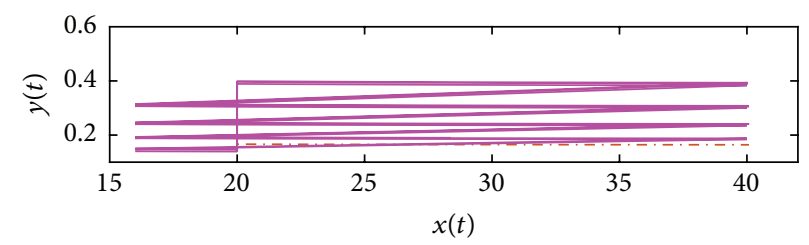

(c)

FIGURE 10: The change in prey density (a), predator density (b), and phase diagrams (c) starting from $\left(x_{0}, y_{0}\right)=(16,0.14)$ with $p=0.6$, $q=0.2, h_{1}=20, h_{2}=40, y_{\tau}\left(h_{1}\right)=0.1664$, and $\alpha_{h_{1}}=0.25$.

considered at different thresholds. At the early stage of the pest damage outbreaks, the biological control is adopted in case of the predator density in the environment lower than its maintainable level. Once the pest density reaches a critical level, the chemical control with a given strength is taken since higher pest density may cause a serious damage to environment. Different to the models in literature, the proposed model is more consistent with practice.

The theoretical analysis indicated that the yield of releases of the predator plays a key role in determining the existence of order-1 periodic orbit. The practical significance to studying the existence of order- 1 or order- 2 periodic solution lies in that it could provide a possibility to determine the frequency of using chemical pesticide and yield of releases of the predator, which makes the control a periodic one without realtime monitoring of the species while keeping the prey density below the damage level. The stability and attractiveness could ensure a certain robustness of control; that is, even though the species density is detected inaccurately or with a deviation, the system will be eventually stable at the periodic solution under the control action.

\section{Competing Interests}

The authors declare that there is no conflict of interests.

\section{Acknowledgments}

This work was supported in part by the National Natural Science Foundation of China (nos. 11401068 and 61473327) and the Liaoning Province Natural Science Foundation of China (no. 2014020133).

\section{References}

[1] J. C. Van Lenteren, "Integrated pest management in protected crops," in Integrated Pest Management, D. Dent, Ed., pp. 311-320, Chapman \& Hall, London, UK, 1995.
[2] J. C. Van Lenteren, "Environmental manipulation advantageous to natural enemies of pests," in Integrated Pest Management, V. Delucchi, Ed., pp. 123-166, Parasitis, Geneva, Switzerland, 1987.

[3] S. Y. Tang and L. S. Chen, "Modelling and analysis of integrated pest management strategy," Discrete and Continuous Dynamical Systems Series B, vol. 4, no. 3, pp. 759-768, 2004.

[4] S. Y. Tang and R. A. Cheke, "State-dependent impulsive models of integrated pest management (IPM) strategies and their dynamic consequences," Journal of Mathematical Biology, vol. 50, no. 3, pp. 257-292, 2005.

[5] S. Y. Tang, Y. N. Xiao, L. S. Chen, and R. A. Cheke, "Integrated pest management models and their dynamical behaviour," Bulletin of Mathematical Biology, vol. 67, no. 1, pp. 115-135, 2005.

[6] L. S. Chen, "The geometry theory of integrated pest management and semi-continuous dynamic system," Journal of Beihua University (Natural Science), vol. 1, pp. 1-9, 2011.

[7] L. Mailleret and F. Grognard, "Global stability and optimisation of a general impulsive biological control model," Mathematical Biosciences, vol. 221, no. 2, pp. 91-100, 2009.

[8] S. Nundloll, L. Mailleret, and F. Grognard, "Two models of interfering predators in impulsive biological control," Journal of Biological Dynamics, vol. 4, no. 1, pp. 102-114, 2010.

[9] A. J. Terry, "Biocontrol in an impulsive predator-prey model," Mathematical Biosciences, vol. 256, pp. 102-115, 2014.

[10] L. Wang, L. Chen, and J. J. Nieto, "The dynamics of an epidemic model for pest control with impulsive effect," Nonlinear Analysis: Real World Applications, vol. 11, no. 3, pp. 1374-1386, 2010.

[11] R. Shi, X. Jiang, and L. Chen, "A predator-prey model with disease in the prey and two impulses for integrated pest management," Applied Mathematical Modelling, vol. 33, no. 5, pp. 22482256, 2009.

[12] R. Q. Shi and L. S. Chen, "An impulsive predator-prey model with disease in the prey for integrated pest management," Communications in Nonlinear Science and Numerical Simulation, vol. 15, no. 2, pp. 421-429, 2010.

[13] H. Zhang, L. S. Chen, and P. Georgescu, "Impulsive control strategies for pest management," Journal of Biological Systems, vol. 15, no. 2, pp. 235-260, 2007.

[14] H. Zhang, P. Georgescu, and L. S. Chen, "On the impulsive controllability and bifurcation of a predator-pest model of IPM," BioSystems, vol. 93, no. 3, pp. 151-171, 2008. 
[15] S. Y. Tang, G. Tang, and R. A. Cheke, "Optimum timing for integrated pest management: modelling rates of pesticide application and natural enemy releases," Journal of Theoretical Biology, vol. 264, no. 2, pp. 623-638, 2010.

[16] B. Liu, Y. J. Zhang, and L. S. Chen, "The dynamical behaviors of a Lotka-Volterra predator-prey model concerning integrated pest management," Nonlinear Analysis: Real World Applications, vol. 6, no. 2, pp. 227-243, 2005.

[17] S. Y. Tang, J. H. Liang, Y. N. Xiao, and R. A. Cheke, "Sliding bifurcations of Filippov two stage pest control models with economic thresholds," SIAM Journal on Applied Mathematics, vol. 72, no. 4, pp. 1061-1080, 2012.

[18] S. Tang, W. Pang, R. A. Cheke, and J. Wu, "Global dynamics of a state-dependent feedback control system," Advances in Difference Equations, vol. 2015, no. 1, article 322, 2015.

[19] S.-Y. Tang, B. Tang, A.-L. Wang, and Y.-N. Xiao, "Holling II predator-prey impulsive semi-dynamic model with complex Poincaré map," Nonlinear Dynamics, vol. 81, no. 3, pp. 15751596, 2015.

[20] G. R. Jiang, Q. S. Lu, and L. N. Qian, "Complex dynamics of a Holling type II prey-predator system with state feedback control," Chaos, Solitons \& Fractals, vol. 31, no. 2, pp. 448-461, 2007.

[21] Y. Tian, K. B. Sun, and L. S. Chen, "Geometric approach to the stability analysis of the periodic solution in a semi-continuous dynamic system," International Journal of Biomathematics, vol. 7, no. 2, Article ID 1450018, 2014.

[22] K. B. Sun, T. H. Zhang, and Y. Tian, "Theoretical study and control optimization of an integrated pest management predatorprey model with power growth rate," Mathematical Biosciences, vol. 279, pp. 13-26, 2016.

[23] L. F. Nie, J. G. Peng, Z. D. Teng, and L. Hu, "Existence and stability of periodic solution of a Lotka-Volterra predator-prey model with state dependent impulsive effects," Journal of Computational and Applied Mathematics, vol. 224, no. 2, pp. 544$555,2009$.

[24] L. Nie, Z. Teng, L. Hu, and J. Peng, "Existence and stability of periodic solution of a predator-prey model with statedependent impulsive effects," Mathematics and Computers in Simulation, vol. 79, no. 7, pp. 2122-2134, 2009.

[25] Y. Tian, K. B. Sun, and L. S. Chen, "Comment on: existence and stability of periodic solu-tion of a Lotka-Volterra predatorprey model with state dependent impulsive effects," Journal of Computational and Applied Mathematics, vol. 234, no. 10, pp. 2916-2923, 2010.

[26] Y. Tian, K.-B. Sun, and L.-S. Chen, "Modelling and qualitative analysis of a predator-prey system with state-dependent impulsive effects," Mathematics and Computers in Simulation, vol. 82, no. 2, pp. 318-331, 2011.

[27] L. C. Zhao, L. S. Chen, and Q. L. Zhang, "The geometrical analysis of a predator-prey model with two state impulses," Mathematical Biosciences, vol. 238, no. 2, pp. 55-64, 2012.

[28] T. Q. Zhang, X. Z. Meng, R. Liu, and T. H. Zhang, "Periodic solution of a pest management Gompertz model with impulsive state feedback control," Nonlinear Dynamics, vol. 78, no. 2, pp. 921-938, 2014. 


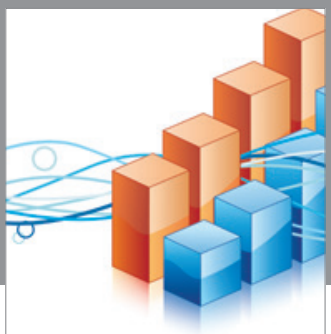

Advances in

Operations Research

vatem alat4

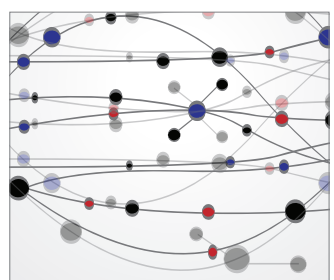

\section{The Scientific} World Journal
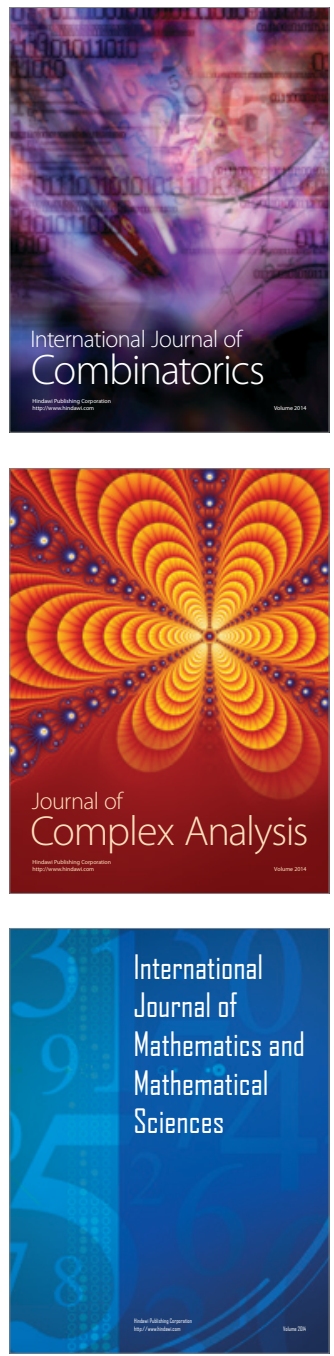
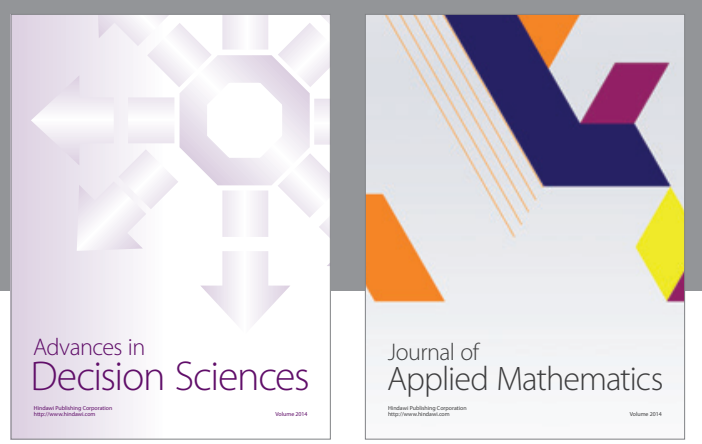

Algebra

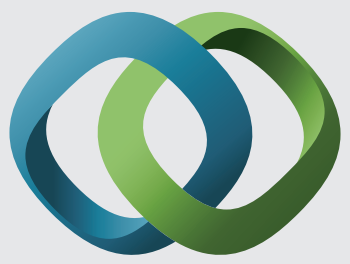

\section{Hindawi}

Submit your manuscripts at

http://www.hindawi.com
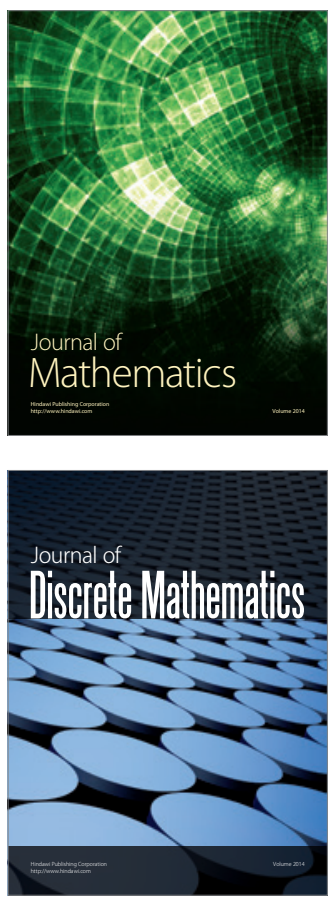

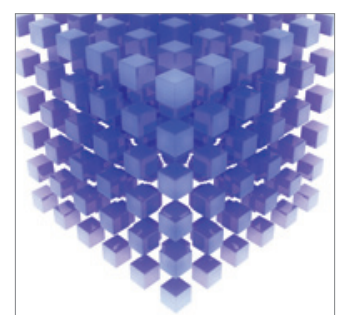

Mathematical Problems in Engineering
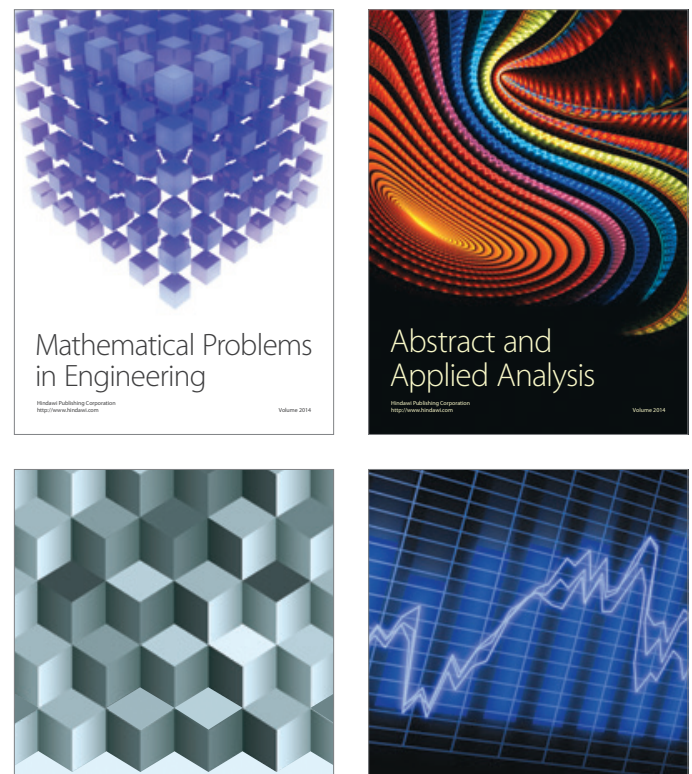

Journal of

Function Spaces

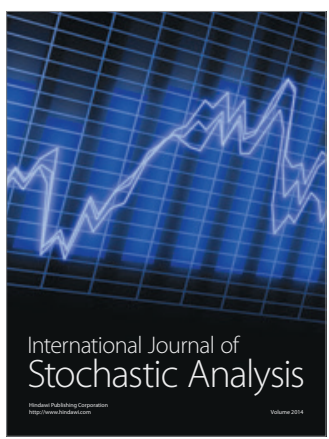

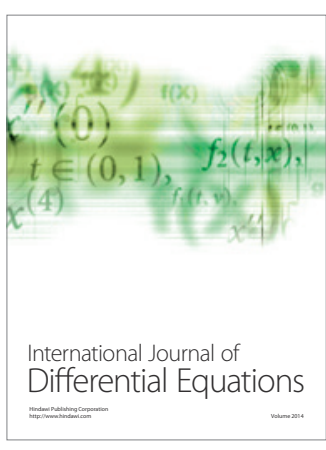
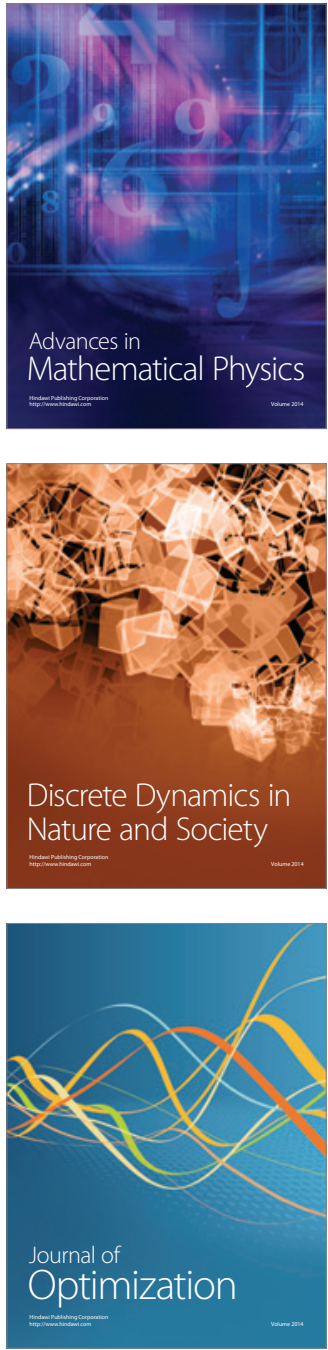